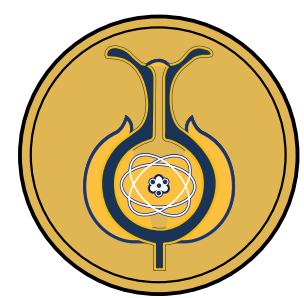

\title{
Conocimiento de profesores de matemáticas en formación sobre los productos notables
}

Knowledge of prospective mathematics teachers on notable products

Conhecimento dos professores de matemáticas em formação sobre os produtos notáveis

\section{Judith Graciano-Barragan ${ }^{1}$ Lilia P. Aké}

Received: Jan/7/2020 • Accepted: Mar/27/2020 • Published: Jan/31/2021.

\section{Resumen}

La presente investigación indaga sobre el conocimiento de futuros profesores de matemáticas en relación con los productos notables. El estudio de corte cualitativo y descriptivo se desarrolla con estudiantes universitarios que cursan un programa de formación de profesores de matemáticas para el nivel bachillerato. Se utilizaron criterios de análisis relativos al Conocimiento Matemático para la Enseñanza como elementos teóricos que permitieron, a partir de la aplicación de un cuestionario, analizar los conocimientos manifestados por los profesores en formación. Se proporciona evidencia de la actividad matemática que realizan los estudiantes para profesor, al resolver tareas que involucran a los productos notables y su enseñanza. Los resultados informan de las inconsistencias en los conocimientos de los futuros docentes y sobre las fortalezas que manifiestan en los subdominios del Conocimiento Matemático para la Enseñanza, lo que permite establecer áreas de desarrollo en su formación.

Palabras clave: conocimiento matemático para la enseñanza; formación de profesores; producto notable; educación matemática; futuros docentes; conocimiento matemático

\section{Abstract}

This paper studies preservice math teachers' knowledge regarding notable products. The qualitative and descriptive study is conducted with university students in a program for high school mathematics teachers. The theoretical elements used were analysis criteria related to Mathematical Knowledge for Teaching. This theoretical foundation allowed the analysis of the preservice teachers' knowledge through a questionnaire. The paper presents evidence of the mathematical activity of preservice teachers when solving tasks and lessons involving notable products. Results report the inconsistencies in preservice teachers' knowledge and the strengths that they manifest in the subdomains of Mathematical Knowledge for Teaching. These results suggest areas of development necessary for teacher training.

Judith Graciano-Barragan, $\triangle$ judith.graciano1c@gmail.com, ㄴ https://orcid.org/0000-0002-5863-1927

Lilia P. Aké,》lake86@gmail.com, @ https://orcid.org/0000-0003-4303-4895

1 Escuela Secundaria General 46 "Ignacio Zaragoza”, Secretaría de Educación Pública, Jalisco, México

2 Facultad de Ingeniería, Universidad Autónoma de Querétaro, Santiago de Querétaro, México 
Keywords: mathematical knowledge for teaching; mathematics education; teacher training; notable product; preservice teachers; knowledge of mathematics

\section{Resumo}

Esta pesquisa investiga o conhecimento de futu ros professores de matemática em relação a produtos notáveis. 0 estudo qualitativo e descritivo é realizado com estudantes universitários que cursam um programa de formação de professores de matemática para o nível de bacharelado. Foram utilizados critérios de análise relacionados ao Conhecimento Matemático para o Ensino como elementos teóricos que permitiram, a partir da aplicação de um questionário, analisar o conhecimento manifestado pelos professores em formação. É fornecida evidência da atividade matemática que os alunos para professor realizam ao resolver tarefas que envolvem produtos notáveis e seu ensino. Os resultados informam as inconsistências no conhecimento de futuros professores e sobre as fortalezas que manifestam nos subdomínios do Conhecimento Matemático para o Ensino, o que permite estabelecer áreas de desenvolvimento em sua formação.

Palavras-chave: conhecimento matemático para o ensino; formação de professores; produto notável; educação matemática; futuros professores; conhecimentos de matemática

\section{INTRODUCCIÓN}

La formación de profesores de matemáticas es una asignatura pendiente entre los investigadores en Didáctica de la Matemática debido a la complejidad que representa definir una ruta formativa inicial y continua para el profesorado de todos los niveles educativos. Particularmente, en el contexto mexicano la formación de quienes enseñan matemáticas en los diferentes niveles educativos de preescolar (3-6 años), primaria (6-12 años), secundaria (12-15 años) y bachillerato (15-18 años) no es homogéneo.

La formación de los maestros de la educación básica (preescolar, primaria y secundaria) es atendida primordialmente, por la Escuela Normal para Maestros, la cual posee el estatus de instituciones de educación superior universitaria (Navarrete-Cazales, 2015). Sin embargo, dicha formación es generalista para los maestros de preescolar y de primaria o de especialización en el caso de los maestros de secundaria. Los elementos formativos que integran sus planes y programas de estudio no profundizan en el conocimiento matemático escolar, no al menos en el sentido propuesto por Ma (1999) y Tzur (2018), quienes indican que la finalización exitosa de los estudios universitarios no es evidencia de una comprensión completa de las matemáticas para ser enseñadas, pues esta acción requiere un tipo apropiado de comprensión que Ma (1999) denomina un conocimiento profundo de las matemáticas que se enseñan.

La formación de los profesores de bachillerato tiene una connotación similar, la mayoría de estos son profesionales egresados de las universidades, aunque cuentan con un conocimiento especializado en su área de formación, no necesariamente, es el requerido para enseñar matemáticas. La mayoría de los profesores de bachillerato en México son ingenieros, contadores, arquitectos, etc., por lo tanto, su conocimiento matemático es situacional y contextual a la profesión para la cual fueron formados. Asimismo, los planes y los programas de formación de estos profesionales no contemplan aspectos teóricos y metodológicos que supone la enseñanza de las matemáticas. 
Un segundo aspecto característico de la formación del profesorado de matemáticas en México es que existen investigaciones realizadas en el área de la formación del profesorado de matemáticas desde la Matemática Educativa o Didáctica de la Matemática (según su lugar de origen), pero las propuestas no son del todo consideradas para la elaboración de planes de estudios de las licenciaturas y posgrados orientadas a la formación de profesores en México (Dolores, 2013). Lo previo contrasta con lo que sucede en otros países, en donde para ejercer la docencia se precisa de realizar algún tipo de estudio adicional, posgrado o especialización (Gómez-Chacón, 2005; Vega-Gil, 2005); en México esto es opcional.

Bajo el contexto descrito surge la necesidad de realizar estudios sobre contenidos matemáticos específicos que permitan entender el conocimiento que los profesores de matemáticas necesitan (Wu, 2018) y los elementos que tienen injerencia en la enseñanza para articular rutas formativas para el profesorado. Particularmente, en este estudio se indaga sobre la enseñanza de los productos notables, debido a la difícil comprensión que tienen los estudiantes sobre esta noción y que resulta una barrera que limita los estudios posteriores (Chang y Tsai, 2005), relacionados con las simplificaciones de fracciones algebraicas y el cálculo de límites aparentemente indeterminados (Vega, 2013) por citar algunos ejemplos.

La enseñanza de este contenido matemático tampoco resulta fácil para los docentes, debido a que típicamente se introduce y se desarrolla de forma mecánica y exigiendo la memorización de la fórmula, sin contemplar, por ejemplo, las interpretaciones geométricas del tema (López, 2008). En este sentido, se precisa establecer una sinergia entre los fines de la educación matemática para los estudiantes y los elementos formativos que necesita el profesor para alcanzar los aprendizajes esperados. Lo previo es importante porque las matemáticas que aprenden los estudiantes y el cómo las aprenden depende de la enseñanza que reciben por parte del profesor (Hill, Rowan y Ball, 2005; Ball, Lubienski y Mewborn, 2001).

Como parte de una de serie de investigaciones relacionadas con la formación de profesores de matemáticas y la enseñanza de los productos notables (Graciano y Aké, 2017; 2019), el presente estudio indaga sobre la siguiente cuestión: ¿cuál es el conocimiento que poseen futuros profesores de matemáticas sobre los productos notables? Para atender la pregunta previa, se utilizó como referente teórico el modelo de Conocimiento Matemático para la Enseñanza (Mathematical Knowledge for Teaching, MKT por sus siglas en inglés), descrito en la sección dos. En una tercera sección se describen las características de la metodología del estudio. En la sección cuatro se evidencia los resultados encontrados, y se finaliza con las conclusiones.

\section{MARCO TEÓRICO}

La investigación toma como elemento teórico el concepto de Conocimiento Matemático para la Enseñanza (MKT de aquí en adelante), el cual es definido como "el conocimiento matemático que utiliza el profesor en el salón de clases para producir instrucción y crecimiento en el aula" (Hill, Ball y Schilling, 2008, p. 374). El MKT está conformado en dos categorías, el conocimiento del contenido y el conocimiento pedagógico del contenido tal y como se observa en la figura 1 .

Como se aprecia en la figura el conocimiento del contenido (SMK), se encuentra dividido en tres subdominios: el 
conocimiento común del contenido matemático, conocimiento especializado del contenido matemático y el conocimiento en el horizonte matemático. Por otra parte, el conocimiento pedagógico del contenido (PCK) se divide en: conocimiento del contenido y los estudiantes, conocimiento del contenido y la enseñanza y, finalmente el conocimiento del currículum. Es importante mencionar que para cuestiones de la investigación y del estatus formativo de la muestra en cuestión se seleccionaron cuatro subdominios, dos de cada una de las categorías

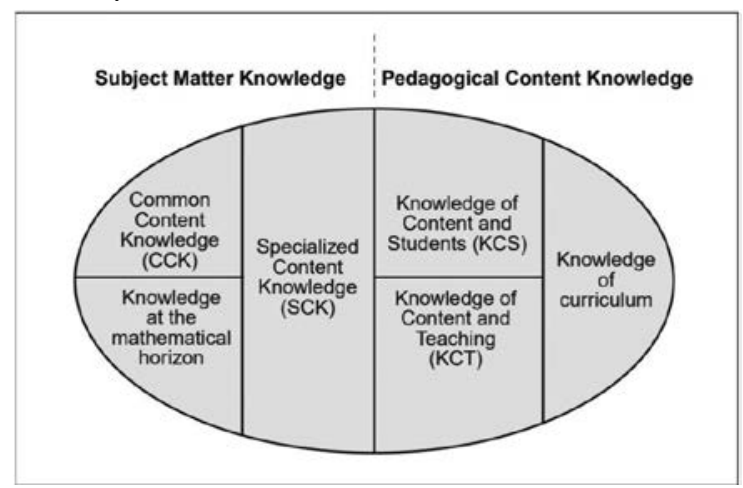

Figura 1. Conocimiento matemático para la enseñanza. Extraído de Hill, Ball y Schilling (2008).

De la primera categoría, se seleccionó el CCK y el SCK. Mientras que, de la segunda categoría, se eligió el KCS y el KCT. A continuación, se describe cada uno de los subdominios utilizados en reportes parciales previos (Graciano y Aké, 2017; 2019).

Conocimiento común del contenido (CCK): este tipo de conocimiento refiere al conocimiento matemático general que poseen las personas, que es común en otras profesiones (Hill et al., 2008) y que permite resolver tareas matemáticas escolares que se asignan a los estudiantes (Ball, Thames y Phelps, 2008; Godino, 2009).
Conocimiento especializado del contenido (SCK): este tipo de conocimiento refiere a las particularidades de la profesión docente. Involucra un conocimiento sobre explicaciones, justificaciones, validaciones y demostraciones a procedimientos y reglas matemáticas utilizadas en las tareas y problemas escolares (Hill et al., 2008). También, implica reconocer y utilizar diferentes representaciones, procedimientos y propiedades de los conceptos matemáticos implicados en la enseñanza (Godino, 2009).

Conocimiento del contenido y los estudiantes (KCS): este tipo de conocimiento hace referencia al proceso de aprendizaje matemático que tienen los estudiantes. Implica que permite explicar el razonamiento de ellos ante la resolución de tareas y problemas (Hill et al., 2008) y sobre los errores, las dificultades y los conflictos de aprendizaje que tiene los alumnos respecto a contenidos matemáticos específicos (Godino 2009; Sosa, 2011).

Conocimiento del contenido y la enseñanza $(\mathrm{KCT})$ : este tipo de conocimiento involucra un conocimiento del proceso de instrucción para la enseñanza (Ball, et al., 2008). Implica la gestión y la planeación de los objetos matemáticos de enseñanza, diferenciar tareas, problemas y recursos más adecuados para el proceso instruccional (Godino, 2009).

\section{METODOLOGÍA}

La investigación tiene un enfoque cualitativo de tipo descriptivo (Creswell, 2009). Se focaliza en el estudio de los aspectos parciales del conocimiento de los productos notables que tienen los profesores, utilizando el modelo MKT. La selección de la muestra es intencional, por tanto, no aleatoria (León y Montero, 2003), ya que 
se eligió al grupo de quinto semestre (de un total de ocho semestres) de un programa universitario de formación de profesores, con un total de 30 estudiantes. Dicha elección estuvo sujeta a la disposición de los alumnos para participar en la investigación.

El estudio implicó dos fases. En la primera se aplicó un cuestionario de respuesta abierta con 6 tareas como instrumento de recolección de dados (ver Tabla 1). En la segunda se realizaron sesiones de 50 minutos de clase bajo el enfoque de la microenseñanza.

Tabla 1. Lógica de la investigación

\begin{tabular}{ll}
\hline & Fase 1 del estudio \\
\hline Instrumento & Cuestionario \\
Técnica & Registro en papel \\
Criterio de análisis & CCK \\
& SCK \\
& KCS \\
& KCT \\
\hline
\end{tabular}

Nota: fuente propia de la investigación.

En el presente artículo se reporta la primera fase del estudio. A través del cuestionario se realizó el análisis de 4 subdominios pertenecientes al MKT, estos son: conocimiento común del contenido (CCK), el conocimiento especializado del contendido (SCK), el conocimiento del contenido y los estudiantes (KCS) y, finalmente, el conocimiento del contenido y la enseñanza (KCT).

A continuación, se muestra en la Tabla 2 la organización de las tareas según el tipo de conocimiento que se estudia.
Las tareas recogidas en el cuestionario son de elaboración propia (ver figura 2). Para la articulación de cada una de las preguntas incluidas en él se consideró la definición de los subdominios previamente abordado en el marco teórico.

\section{ANÁLISIS Y RESULTADOS}

A continuación, se desarrollan los resultados encontrados por cada dominio del conocimiento del MKT a partir del cuestionario. Las descripciones y los análisis realizado con base en los criterios de análisis del MKT, permitieron discernir entre las respuestas dadas por los futuros maestros y categorizar su conocimiento. Para esta categorización se designaron las etiquetas de en vías de desarrollo, parcialmente desarrollado y desarrollado (Graciano y Aké, 2017).

\section{Sobre el conocimiento común (CCK)}

Como se hizo mención en el apartado del Marco Teórico, el Conocimiento Común del Contenido (CCK, por sus siglas en inglés), refiere al conocimiento que poseen las personas a modo general, que es común en otras profesiones y que permite resolver tareas matemáticas escolares que se asignan a los estudiantes. Este tipo de conocimiento se indagó con las tareas 1 y 2 .

La tarea 1 de simplificaciones de expresiones algebraicas está integrada por los ítems

Tabla 2. Distribución de los tipos de conocimiento analizados en la fase 1 del estudio

\begin{tabular}{|c|c|c|c|c|c|c|c|c|c|c|c|c|c|c|c|}
\hline \multirow{4}{*}{ Conocimiento } & \multicolumn{15}{|c|}{ Fase 1} \\
\hline & \multirow{2}{*}{\multicolumn{2}{|c|}{ T1 }} & & & \multicolumn{2}{|l|}{$\mathbf{T} 2$} & \multicolumn{2}{|c|}{ T3 } & \multirow{2}{*}{\multicolumn{2}{|c|}{ T4 }} & \multicolumn{2}{|c|}{ T5 } & \multicolumn{3}{|c|}{ T6 } \\
\hline & & & Cor & nas & & & & & & & nsi & & & & \\
\hline & $\mathrm{a}$ & $\mathrm{b}$ & $\mathrm{c}$ & $\mathrm{a}$ & $\mathrm{b}$ & $\mathrm{c}$ & a & $\mathrm{b}$ & $\mathrm{a}$ & & $\mathrm{a}$ & $\mathrm{b}$ & $\mathrm{a}$ & $\mathrm{b}$ & $\mathrm{c}$ \\
\hline cck & $\mathrm{x}$ & $\mathrm{x}$ & $\mathrm{x}$ & $\mathrm{x}$ & $\mathrm{x}$ & $\mathrm{x}$ & & & & & & & & & \\
\hline sck & & & & & & & & & $x$ & & & $\mathrm{x}$ & $\mathrm{x}$ & & \\
\hline kes & & & & & & & $\mathrm{x}$ & & & & $\mathrm{x}$ & & & & $\mathrm{x}$ \\
\hline $\mathrm{kct}$ & & & & & & & & $\mathrm{x}$ & & & & & & $\mathrm{x}$ & \\
\hline
\end{tabular}




\begin{tabular}{|c|c|}
\hline $\begin{array}{l}\text { Tarea 1 } \\
\text { Simplifica las siguientes expresiones } \\
\text { algebraicas }\end{array}$ & $\begin{array}{l}\text { Tarea } 2 \\
\text { Calcula los siguientes límites de manera } \\
\text { algebraica }\end{array}$ \\
\hline a) $\frac{x^{2}-14 x+49}{(x-7)^{2}(x-7)}$ & $\begin{array}{l}\text { a) } \lim _{x \rightarrow 7} \frac{\left(x^{2}+4 x+4\right)(x-2)}{x^{2}-4} \\
\text { b) } \lim _{x \rightarrow 3} \frac{3(x+3)(x-3)}{2\left(x^{2}-9\right)}\end{array}$ \\
\hline $\begin{array}{l}\text { b) } \frac{\left(4 x^{2}-1\right)\left(4 x^{2}+1\right)}{(2 x+1)(2 x-1)} \\
\text { c) } \frac{(3 x+9)^{2}(x+9)}{27 x^{2}+243 x^{2}+729 x+729}\end{array}$ & c) $\lim _{x \rightarrow-9} \frac{3(x+9)}{x^{2}-4 x-117}$ \\
\hline $\begin{array}{l}\text { Tarea } 3 \\
\text { En clase el profesor propuso los siguientes } \\
\text { ejercicios a sus alumnos. } \\
\text { - }(x+7)(x-7) \\
\text { - }(x+7)(x+7) \\
\text { Para lo cual uno de sus alumnos realiza el } \\
\text { siguiente procedimiento y da respuesta a los } \\
\text { ejercicios. }\end{array}$ & $\begin{array}{l}\text { Tarea } 4 \\
\text { Un alumno resuelve la simplificación de } \\
\text { fracciones desarrollando la división expresada, } \\
\text { como se muestra a continuación: } \\
\frac{9 x^{2}-48 x+72}{3 x-8}\end{array}$ \\
\hline$\left.\bigcap^{+17)(x-7)-x^{2}-49} \quad\right|^{(x+7)(x+7)=} x^{2}+49$ & $\begin{array}{l}\text { a) ¿Es viable utilizar siempre división? ¿Por } \\
\text { qué? }\end{array}$ \\
\hline $\begin{array}{l}x^{2}(\bullet x-19 \\
\text { a) Explica el razonamiento que pudo seguir el } \\
\text { alumno para plantear sus respuestas. } \\
\text { b) ¿Cómo le explicarias al alumno el tema y la } \\
\text { formalización del algoritmo de binomios } \\
\text { conjugados? }\end{array}$ & $\begin{array}{l}\text { b) ¿Cómo le explicarías al alumno la } \\
\text { simplificación de fracciones en casos donde } \\
\text { se involucren productos notables? }\end{array}$ \\
\hline $\begin{array}{l}\text { Tarea } 5 \\
\text { En la clase, el profesor dejó un ejercicio el cual } \\
\text { un alumno resolvió de la siguiente manera: } \\
\qquad(a-b)^{3}=a^{3}-3 a^{2} b^{2}+3 a b-b^{3}\end{array}$ & $\begin{array}{l}\text { Tarea } 6 \\
\text { Analiza el siguiente ejercicio aritmético que } \\
\text { involucra productos notables: } \\
\qquad(12+6)^{2}=324\end{array}$ \\
\hline $\begin{array}{l}\text { a) Explica el posible razonamiento que } \\
\text { condujo al alumno a dar esa respuesta. } \\
\text { b) Identifica los conceptos de tipo algebraico } \\
\text { que se ponen en juego al resolver este } \\
\text { ejercicio. }\end{array}$ & $\begin{array}{l}\text { a) ¿Cómo sabes que el resultado es correcto? } \\
\text { b) ¿El ejercicio sería un ejemplo recomendable } \\
\text { para los alumnos al inicio del tema de } \\
\text { productos notables? ¿Por qué? } \\
\text { c) ¿Qué errores podrian cometer los alumnos a } \\
\text { partir del ejercicio? }\end{array}$ \\
\hline
\end{tabular}

Figura 2. Tareas del cuestionario.

a, b y c. La tarea 2 de cálculo de límites aparentemente indeterminados, también está integrada por tres ítems, a, b y c. Para resolver estas tareas, el futuro profesor tiene que identificar el producto notable, sin la necesidad de realizar la multiplicación indicada en las expresiones. El análisis de las respuestas dadas permitió la categorización presentada en la Tabla 3.

Tabla 3. Estatus del conocimiento común

\begin{tabular}{|c|c|c|c|c|c|c|c|}
\hline \multirow[t]{2}{*}{ Estatus } & \multirow[t]{2}{*}{ Descriptor } & \multicolumn{6}{|c|}{$\begin{array}{c}\text { Número de profesores por cada tarea } y \\
\text { consigna }\end{array}$} \\
\hline & & T1a & T1b & T1c & $\mathbf{T} 2 \mathbf{a}$ & $\mathbf{T} 2 \mathbf{b}$ & T2c \\
\hline Desarrollado & $\begin{array}{l}\text { Identifica el producto notable y realiza la } \\
\text { simplificación de fracciones o el cálculo } \\
\text { del límite aparentemente indeterminado } \\
\text { de manera correcta. }\end{array}$ & 23 & 14 & 20 & 11 & 12 & 10 \\
\hline
\end{tabular}




\begin{tabular}{|c|c|c|c|c|c|c|c|}
\hline \multirow[t]{2}{*}{ Estatus } & \multirow[t]{2}{*}{ Descriptor } & \multicolumn{6}{|c|}{$\begin{array}{c}\text { Número de profesores por cada tarea y } \\
\text { consigna }\end{array}$} \\
\hline & & T1a & T1b & T1c & T2a & T2b & T2c \\
\hline $\begin{array}{l}\text { Parcialmente } \\
\text { desarrollado }\end{array}$ & $\begin{array}{l}\text { No identifica el producto notable, pero } \\
\text { determina la respuesta correcta, dado que } \\
\text { se desarrolla las multiplicaciones indica- } \\
\text { das para comprobar si efectivamente es } \\
\text { posible realizar una simplificación. }\end{array}$ & 2 & 7 & 5 & 0 & 1 & 0 \\
\hline $\begin{array}{l}\text { En vías de de- } \\
\text { sarrollo }\end{array}$ & $\begin{array}{l}\text { No identifica el producto notable y pre- } \\
\text { senta errores que no permiten llegar a la } \\
\text { resolución de la tarea. }\end{array}$ & 5 & 4 & 4 & 18 & 14 & 15 \\
\hline No desarrollado & & 0 & 1 & 1 & 1 & 3 & 5 \\
\hline
\end{tabular}

En la tarea 1, el ítem $a$ fue resuelto de manera correcta por 25 de los 30 estudiantes que participaron en el estudio. De manera similar el ítem $c$, también fue resuelto de manera satisfactoria por la mayoría de los futuros profesores. En el caso del ítem $b, 21$ profesores en formación respondieron de forma acertada, pero 7 de ellos luego de llegar a la respuesta comprobaron su resultado realizando la multiplicación.

Por ejemplo, en la figura 3 se muestra un caso específico del futuro profesor 2 en el que se realiza la multiplicación del binomio al cuadrado $(x-7)^{2}=(x-7)(x-7)$. Con la operación anterior el estudiante para profesor comprueba que la expresión del numerador $x^{2}-14 x+49$ es igual a $(x-7)^{2}$ y cancela los términos.

La tarea 2 que involucra el cálculo de límites aparentemente indeterminados resultó ser difícil para los profesores en formación. Los futuros profesores no
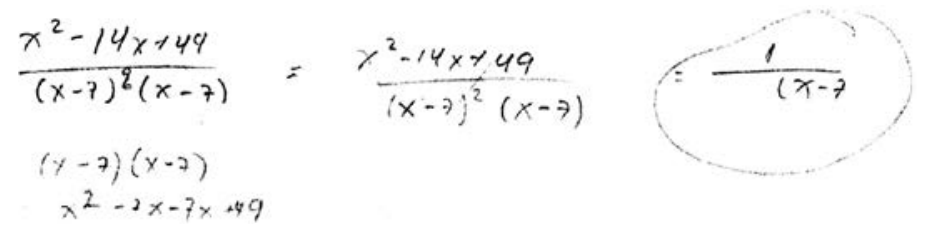

Figura 3. Evidencia del FP2 sobre su conocimiento común parcialmente desarrollado asocian el uso de los productos notables para "eliminar" la indeterminación de la expresión y realizan el cálculo del límite como si se tratase de una función continua. Como ejemplo se muestra la actividad matemática del estudiante para profesor PF5 para el ítem $c$.

$$
\begin{aligned}
\lim _{x \rightarrow-9} & \frac{3(x+9)}{x^{2}-4 x-117} \\
& =\frac{3(-9+9)}{(-9)^{2}-4(-9)-117} \\
& =\frac{3(0)}{81+36-117} \\
& =\frac{0}{0} \text { Indeterminacio }
\end{aligned}
$$

Figura 4. Evidencia del FP5 sobre su conocimiento común en vías de desarrollo

De manera general, este Conocimiento Común del Contenido (CCK) se encuentra limitado en los estudiantes para profesor, debido a que en situaciones descontextualizadas son capaces de reconocer el producto notable (aunque en 
algunos casos necesiten realizar la comprobación), pero en otros contextos de aplicación presentaron errores no solo en el reconocimiento de la estructura del producto notable, sino también en este caso, en la resolución de los límites aparentemente indeterminados.

\section{Sobre el conocimiento especializado (SCK)}

En el marco teórico se expresó que el Conocimiento Especializado del Contenido (SCK) es aquel que refiere a las particularidades de la profesión docente. Este tipo de conocimiento se indagó con las tareas 4,5 y 6 en sus ítems $a, b$ y $a$, respectivamente. La tarea 4 plantea una simplificación de fracciones algebraicas y se propone una solución por división; se cuestiona al futuro profesor sobre la pertinencia de este método de división para abordar la solución a la simplificación de fracciones algebraicas. La tarea 5 plantea una posible solución de un estudiante al desarrollo de un binomio al cubo y se cuestiona al profesor en formación sobre los objetos algebraicos involucrados en la resolución. Por otro lado, la tarea 6 plantea la respuesta de un producto notable en un contexto, al futuro profesor se le cuestiona sobre la validez del procedimiento. El análisis de las respuestas dadas por los estudiantes se presenta a continuación en la Tabla 4.

Tabla 4. Estatus del conocimiento especializado

\begin{tabular}{|c|c|c|c|}
\hline Estatus & Descriptor & $\begin{array}{c}\text { Tarea y } \\
\text { consigna }\end{array}$ & $\begin{array}{l}\text { Número de } \\
\text { profesores }\end{array}$ \\
\hline \multirow[t]{3}{*}{ Desarrollado } & $\begin{array}{l}\text { Alude a los métodos que facilitarían a los alumnos la simplifi- } \\
\text { cación de fracciones, idealmente identifica y explica las dificulta- } \\
\text { des y los errores que el método de división puede propiciar. }\end{array}$ & $4 a$ & 15 \\
\hline & $\begin{array}{l}\text { Identifica los conceptos algebraicos que se ponen en manifiesto al } \\
\text { resolver el ejercicio de binomio al cubo. }\end{array}$ & $5 b$ & 5 \\
\hline & $\begin{array}{l}\text { Realiza explicaciones sobre la validez de los procedimientos } \\
\text { empleados en relación con la resolución de la expresión aritmética } \\
\text { que involucra el binomio al cuadrado. }\end{array}$ & $6 a$ & 9 \\
\hline \multirow[t]{3}{*}{$\begin{array}{l}\text { Parcialmente } \\
\text { desarrollado }\end{array}$} & $\begin{array}{l}\text { Efectúa explicaciones inconsistentes sobre los errores que favore- } \\
\text { cería la utilización de la división como método para simplificar } \\
\text { fracciones algebraicas. }\end{array}$ & $4 a$ & 0 \\
\hline & $\begin{array}{l}\text { Identifica algunos de los conceptos empleados en la resolución del } \\
\text { ejercicio de binomio al cubo. }\end{array}$ & $5 b$ & 12 \\
\hline & $\begin{array}{l}\text { Realiza explicaciones sobre la expresión aritmética que involucra } \\
\text { el binomio al cuadrado, pero basándose en procedimientos pura- } \\
\text { mente aritméticos y no en la estructura del producto notable. }\end{array}$ & $6 a$ & 16 \\
\hline \multirow[t]{3}{*}{$\begin{array}{l}\text { En vías de desar- } \\
\text { rollo }\end{array}$} & $\begin{array}{l}\text { Falta de explicaciones que justifiquen la respuesta del futuro } \\
\text { profesor respecto a la simplificación de fracciones utilizando la } \\
\text { operación de división. }\end{array}$ & $4 a$ & 14 \\
\hline & $\begin{array}{l}\text { Falta de identificación y explicación de conceptos algebraicos } \\
\text { plasmados en el ejercicio sobre el binomio cubo. }\end{array}$ & $5 b$ & 13 \\
\hline & $\begin{array}{l}\text { Realiza explicaciones sin identificar la estructura del producto } \\
\text { notable y cometiendo errores en dichas explicaciones. }\end{array}$ & $6 a$ & 5 \\
\hline \multirow[t]{3}{*}{ Sin contestar } & & $4 a$ & 1 \\
\hline & & $5 b$ & 0 \\
\hline & & $6 a$ & 0 \\
\hline
\end{tabular}

Nota: ampliado y modificado de Graciano y Aké (2017; 2019). 
De la Tarea 4 en su ítem $a$, se solicitaba al profesor en formación explicar la pertinencia de utilizar división para la simplificación de fracciones algebraicas. En este caso, de los 15 profesores en formación que cuyo estatus de conocimiento especializado se categorizó como desarrollado, 7 hicieron hincapié en que existen otros métodos que les facilitarían a los alumnos la simplificación de fracciones, un ejemplo de dicha afirmación es la mencionada por el profesor en formación 27 (PF27).

PF27: "Muchas de las veces nos familiarizamos con un método, pero si es necesario que se doten de más formas de resolución pues algunas veces existen formas más fáciles como factorizar y división sintética".

Por otra parte, los otros 8 futuros docentes resaltan las diversas dificultades a las que se pueden enfrentar los alumnos al momento de realizar la división, pero también mencionan sobre los errores que puede generar al realizar de esta forma dicha simplificación (PF6). Por el contrario, 14 de 30 futuros profesores no tienen explicaciones claras, es decir, ofrecen una respuesta confusa para respaldar la viabilidad de utilizar la división (EP 21).

PF6: "Desde mi punto de vista creo que si el alumno no sabe factorizar sería más sencillo para la aplicación de la división; pero en esto existen 3 variantes, a) cuando el alumno no sabe realizar de forma correcta la división tendrá por ende la respuesta errónea, b) cuando el alumno sabe realizarla; pero por alguna razón se le "va" un signo o una operación errónea, lo que causará que su resultado este mal (es lo que sucede, quiero imaginar, en el ejemplo empleado), y c) otro aspecto que puede suceder es cuando existe el caso en el que la división da residuo y el alumno no sabe cómo representarlo, así que creo que puede ser viable la división siempre y cuando no sea tan fácil o no se pueda factorizar una ecuación"

PF21: "Sí es viable ya que da el resultado de la división, que es la mínima expresión".

Para la Tarea 5 en su ítem $b$, se solicitaba al futuro profesor los conceptos algebraicos que se ponen en juego para resolver el ejercicio de binomio al cubo, es decir los conceptos que el alumno debe conocer para resolver dicho ejercicio. En este sentido, 5 futuros docentes identifican relaciones de conceptos (e. g., E12 y PF27).

PF12: "Potencia, multiplicación de signos, productos de monomios, triángulo de pascal y binomio de Newton”.

PF27: "Al realizar este tipo de ejercicios existen varios factores en los cuales es posible cometer errores, por ejemplo: leyes de los exponentes, leyes de los signos, multiplicación, triángulo de pascal, regla del binomio al cubo".

Se aprecia la manifestación de distintos conceptos que pueden ponerse en juego en el planteamiento de la división, aunque no todos son de naturaleza algebraica, lo que indica que no disciernen de las implicaciones aritméticas de las algebraicas, al menos en esta tarea planteada. Por otro lado, 12 de 30 profesores en formación, identifican algunos de los conceptos empleados, pero utilizan un lenguaje que no es el adecuado, ya que usan términos no establecidos para dichos conceptos (e. g., PF30).

Diez futuros docentes no identifican los conceptos algebraicos involucrados, ya 
que las respuestas hacen hincapié a la forma de resolución del ejercicio, posibles errores que se pueden desarrollar, a la manera más sencilla de resolverlo o simplemente los estudiantes resaltan que no sabían conceptos exactos (e. g., PF29).

PF29: "Pueden utilizar diferentes métodos, triángulo de pascal, binomio de newton o dar ejemplos numéricos que le sean significativos a los alumnos"

PF30: El problema es el signo negativo al resolver el procedimiento en la multiplicación de signos

La tarea 6 en su ítem $a$ plantea el producto notable en el contexto aritmético y se propone una respuesta, al futuro profesor se le solicita validar dicho resultado. Al respecto, 9 docentes en formación realizan una correcta explicación en función al contenido de la tarea sobre binomio al cuadrado (PF30). Por otro lado, 16 docentes en formación realizan la explicación únicamente del procedimiento aritmético para comprobar si el resultado del ejercicio es correcto, no aluden al producto notable (PF13).

PF30: "Es correcto al sumar el cuadrado del primero, más el doble del primero por el segundo más el cuadrado del segundo, ya que es un binomio al cuadrado".

PF13: "Es correcto porque puedo sumar lo que hay dentro del paréntesis y solo elevarlo al cuadrado $(12+6)^{2}=(18)^{2}=324$ ".

De manera general se puede observar que el SCK de la mayoría de los profesores en formación se encuentra (entre 13 y 14 profesores) en vías de desarrollo, al ofrecer explicaciones de carácter procedimental sobre el uso de la división para la simplificación de expresiones algebraicas. Además, presentan inconsistencias en las explicaciones e identificaciones sobre conceptos, reglas y/o procedimientos que aluden al binomio al cubo.

Los planteamientos, también ponen de manifiesto que la interpretación del binomio al cuadrado en el plano algebraico (uso de letras) no se concibe que pueda ser desarrollado en contextos aritméticos. Lo previo es congruente con el estudio de Vega (2013), quien menciona que los alumnos difícilmente consideran la relación entre los números y las variables, puesto que no perciben los productos notables en expresiones numéricas.

\section{Conocimiento del contenido mate- mático y los estudiantes (KCS)}

Por otro lado, las tareas 3, 5 y 6 están vinculadas al conocimiento del contenido matemático y los estudiantes en sus ítems $a, a$ y $c$ respectivamente. La tarea 3 plantea un binomio conjugado y un binomio al cuadrado con soluciones hipotéticas, mientras que la tarea 5 involucra también una respuesta hipotética, pero al desarrollo de un binomio al cubo. En ambas tareas se cuestiona al futuro profesor sobre los razonamientos que pudieron dar lugar a las soluciones dadas, se pretende que identifique y explique errores. Por otro lado, la tarea 6 plantea el binomio al cuadrado en el contexto aritmético y se propone una respuesta, al futuro profesor se le solicita expresar los posibles errores que pudiera favorecer el abordar el producto notable de la manera expuesta. En el análisis de las respuestas dadas por los estudiantes para profesor se presenta en la siguiente tabla (ver Tabla 5). 
Tabla 5. Estatus del conocimiento del contenido y los estudiantes

\begin{tabular}{llcc}
\hline \multicolumn{1}{c}{ Estatus } & \multicolumn{1}{c}{ Descriptor } & $\begin{array}{c}\text { Tarea y } \\
\text { consigna }\end{array}$ & $\begin{array}{c}\text { Número de } \\
\text { profesores }\end{array}$ \\
\hline Desarrollado & Predice e identifica errores respecto al contenido de productos & $3 \mathrm{a}$ & 6 \\
& notables. & $5 \mathrm{a}$ & 5 \\
& & $6 \mathrm{c}$ & 12 \\
Parcialmente desar- & Identifica el error más no a la naturaleza de este, las explica- & $3 \mathrm{a}$ & 6 \\
rollado & ciones presentan inconsistencias y se utilizan términos y concep- & $5 \mathrm{a}$ & 4 \\
& tos erróneos. & $6 \mathrm{c}$ & 11 \\
En vías de desar- & No identifica errores, se realizan descripciones del procedimien- & $3 \mathrm{a}$ & 18 \\
rollo & to y se utilizan términos y conceptos inadecuados. & $5 \mathrm{a}$ & 21 \\
& & $6 \mathrm{c}$ & 7 \\
\hline
\end{tabular}

Nota: fuente propia de la investigación.

En la tarea 3, inciso a) se indaga sobre la identificación de las concepciones erróneas que tienen los alumnos respecto al binomio al cuadrado y los binomios conjugados, a partir de la visualización de los procedimientos empleados, es decir, si el futuro profesor identifica los errores al explicar el razonamiento que pudo seguir el alumno para plantear sus respuestas. En este sentido, lo ideal es que los futuros profesores se percaten del error, el cual consiste en resolver del mismo modo el binomio conjugado y binomio al cuadrado.

Al respecto, 6 maestros en formación identifican la concepción errónea de dar por válida la distributiva de la potencia, a partir de la equivalencia de $(a+b)^{2}=(a+b)(a-b)$. En este sentido, los futuros profesores visualizan que el procedimiento utilizado por el alumno no es acertado (PF17). Asimismo, 6 futuros docentes identifican el error en la equivalencia entre $(a+b)^{2}=(a+b)(a-b)$, pero los argumentos establecidos son inconsistentes, además de que los futuros profesores emplean conceptos no establecidos y no utilizan un lenguaje matemático adecuado. Como ejemplo, se expresa la respuesta del futuro profesor 12 , quien no identifica que el error procede de la extrapolación de la estructura del binomio al cuadrado, al de la diferencia de cuadrados (EP12).

PF17: "El alumno resolvió ambos como si fuesen binomios conjugados, para esto falta en el alumno comprender la estructura, así como especificar las diferencias con un binomio al cuadrado $(x+7)^{2}$. Es muy común que con el tema de productos notables los alumnos confundan el algoritmo".

PF12: "El razonamiento del alumno para resolver los binomios conjugados fue elevar al cuadrado y multiplicar signos. Aunque en el binomio $(x+7)(x-7)$ el procedimiento que realizó el alumno si cumplió para el resultado, pero para el binomio $(x+7)(x+7)$ no cumple".

Finalmente, 18 futuros docentes no identifican los errores en el ejercicio, ya que describen el procedimiento utilizado por el alumno sin explicar el error cometido y sin hacer hincapié en los productos notables involucrados. En el siguiente ejemplo se evidencia este hecho en el que el futuro profesor parece no reconocer el error en el planteamiento proporcionado e incluso sugiere que es correcto.

PF14: "El alumno solo elevó al cuadrado el primer término y el segundo. Además, realizó la multiplicación de los signos para saber cuál tenía que poner. Es por eso por lo que en el primer caso colocó una diferencia o resta, y en el segundo caso colocó una suma" 
La tarea 5, en su ítem $a$ exige al futuro profesor identificar y explicar los errores que condujeron a dar dicha respuesta al ejercicio, para analizar los procedimientos y los razonamientos del alumno que condujeron a dar esa respuesta al ejercicio. Al respecto, 5 profesores en formación identifican el error que se expresa en el resultado del binomio al cubo y explican los posibles procedimientos y conceptos erróneos que se emplearon (EP14). Por otra parte, 4 docentes en formación identifican el concepto mal empleado de acuerdo con el error. En este sentido, cabe mencionar que los argumentos no son claros, ya que emplean términos y conceptos erróneos a causa de una asociación errónea entre el concepto y su utilidad (PF6).

PF14: "Quizá utilizó el Binomio de Newton o el triángulo de Pascal para obtener los coeficientes que debería tener cada término, pero se confundió a la hora de colocar los exponentes, ya que en cada término la suma de los exponentes debe ser igual al grado del producto”.

PF6: "Es claro que el alumno utilizó erróneamente el triángulo de pascal ya que no logró comprender como es la distribución de las potencias y esto provoca un resultado erróneo".

Veinte futuros profesores describen el procedimiento del alumno y en algunos casos identifican que la respuesta es incorrecta, pero no saben a qué se debe dicho error. Asimismo, emplean términos y conceptos inadecuados de acuerdo con los usos y los significados. De esta manera, el estudiante 1 expresa:

PF1: "Fue multiplicando los 3 términos $(a-b)(a-b)(a-b)$ pudo a ver multiplicado de uno por uno o con la regla del binomio al cubo, pero no siguió ninguna de los dos, solo fue poniendo cada uno cada bloque la elevación que sigue".

Finalmente, la tarea 6, implica al profesor predecir los errores que los alumnos pueden cometer a partir del ejercicio aritmético, es decir el futuro profesor analiza la diversidad de complicaciones que el alumno puede enfrentar, pero con relación al producto notable involucrado (binomio al cuadrado). Doce futuros profesores identifican las dificultades y los errores respecto al producto notable y explican de qué trata dicho error, aunque dicha explicación se remite a un carácter procedimental (FP30).

Por otro lado, once docentes en formación identifican posibles errores que el alumno puede cometer, pero estos no necesariamente, se involucran con el binomio al cuadrado. Dicho de otra manera, el futuro profesor únicamente resalta los errores que, en lo general, se pueden suscitar en diversos temas, es decir, errores que van desde una suma hasta la confusión de lo que implica elevar al cuadrado (FP10).

PF30: "El error más común es solo elevar los números al cuadrado sin utilizar la regla lo cual les da un resultado incorrecto".

PF10: "Multiplicar por 2 los números dentro del paréntesis o no seguir la jerarquía d operaciones".

Seis futuros docentes no identifican las dificultades y los errores que los alumnos pueden cometer de acuerdo con lo expuesto en el ejercicio o simplemente confunden un procedimiento acertado con uno erróneo, indica que no reconocen la estructura del binomio al cuadrado en el plano aritmético (PF26). Por último, el alumno restante no 
responde a la interrogante del ítem, lo cual indica que dicho futuro profesor no identifica las dificultades y los errores que los alumnos pueden llegar a cometer en el ejercicio.

PF26: "Que solo eleve al cuadrado el 6, que quieran poner la expresión $(12+6)$ $(12+6)$ y multiplicar los números sin antes sumarlos".

De manera general, el KCS es un conocimiento en el que la mayoría de los estudiantes para profesor se encuentran en el estatus de en vías de desarrollo (entre 18 y 21), ya que los futuros profesores tienen inconsistencias en la identificación, la explicación y la predicción de los errores que pueden tener los alumnos sobre el contenido de productos notables. Incluso, se puso de manifiesto interpretaciones erróneas de los futuros docentes, por ejemplo, al dar por válida la distributiva de la potencia, o en aquellas respuestas que no van direccionadas hacia la explicación del procedimiento, para calcular la respuesta planteada en la tarea.

Es importante mencionar que confrontar a los alumnos con sus errores es esencial para la superación de estos; en este sentido los profesores deberían tener un conocimiento sobre los errores para organizar líneas de actuación específicas, para conducir mejor la enseñanza-aprendizaje de las matemáticas, insistiendo en aquellos aspectos que generan más dificultades.

\section{Conocimiento del contenido mate- mático y la enseñanza (KCT)}

El conocimiento del contenido de productos notables y la enseñanza es el conocimiento que implica discriminar, seleccionar, diseñar o rediseñar ejercicios del contenido de productos notables en relación con el momento en que serán presentados, es decir, si dichos ejercicios se utilizarán para comenzar un contenido o para profundizar sobre este (Ball, et al., 2008). Asimismo, comprende el saber qué ejemplos y representaciones son las adecuadas para enseñar el contenido. En este sentido, dicho conocimiento se ve expresado en las tareas 3,4 y 6 en los ítems $b$ de las tres tareas (ver Tabla 6).

Tabla 6. Estatus del conocimiento matemático y la enseñanza

\begin{tabular}{|c|c|c|c|}
\hline Categoría & Descriptor & $\begin{array}{c}\text { Tarea y } \\
\text { consigna }\end{array}$ & $\begin{array}{l}\text { Número de } \\
\text { profesores }\end{array}$ \\
\hline Desarrollado & $\begin{array}{l}\text { Alude a diversas representaciones y/o explicaciones tanto } \\
\text { de carácter conceptual como procedimental para aproximar } \\
\text { la comprensión del tema al alumno. } \\
\text { Discrimina ejemplos para iniciar y profundizar en el tema } \\
\text { de productos notables de acuerdo con las dificultades a las } \\
\text { que el alumno se pueda enfrentar. }\end{array}$ & $\begin{array}{l}3 b \\
4 b \\
6 b\end{array}$ & $\begin{array}{l}0 \\
0 \\
4\end{array}$ \\
\hline $\begin{array}{l}\text { Parcialmente desar- } \\
\text { rollado }\end{array}$ & $\begin{array}{l}\text { Explica en relación con aspectos procedimentales. } \\
\text { Propone ejemplos para iniciar el tema de productos nota- } \\
\text { bles, aunque no discrimina de aquellos que son adecuados } \\
\text { para profundizar. No se considera las dificultades que } \\
\text { pudieran emerger en los alumnos. }\end{array}$ & $\begin{array}{l}3 b \\
4 b \\
6 b\end{array}$ & $\begin{array}{c}23 \\
16 \\
6\end{array}$ \\
\hline $\begin{array}{l}\text { En vías de desar- } \\
\text { rollo }\end{array}$ & $\begin{array}{l}\text { No alude a las representaciones o explicaciones ni de corte } \\
\text { conceptual, ni procedimental para aproximar la compren- } \\
\text { sión del tema al alumno, más bien, se ponen de manifiesto } \\
\text { errores en la interpretación del producto notable. } \\
\text { No propone ejemplos. }\end{array}$ & $\begin{array}{l}3 b \\
4 b \\
6 b\end{array}$ & $\begin{array}{c}7 \\
14 \\
20\end{array}$ \\
\hline
\end{tabular}

Nota: fuente propia de la investigación. 
En la tarea 3, inciso $b$, se pone en manifiesto la explicación del algoritmo de binomios conjugados. Por lo que se refiere a las respuestas de los futuros profesores para dicha tarea, se distinguen tres tipos de explicaciones de carácter procedimental manifestada por 23 futuros docentes. En el primer tipo de respuesta 14 futuros docentes afirman que realizarían una explicación mediante el desarrollo de las multiplicaciones que se expresan en los binomios conjugados, de tal manera que después los alumnos realicen de forma autónoma una generalización (PF11).

PF11: "Le explicaría desarrollando varios ejercicios, pero paso a paso, es decir: $(x+7)(x-7)=x^{2}-7 x+7 x-49=$ $x^{2}-49$. Y con ello lograría que los alumnos deduzcan que en estos casos los términos se elevan al cuadrado y se deja el signo menos. Lo mismo haría para el otro caso".

Se aprecia que la explicación del futuro docente es procedimental; en este caso se podría promover una reflexión en los estudiantes al proporcionar un escenario de confrontación con sus propios errores y llevarse a cabo una explicación por medio de un enfoque geométrico (López, 2008), realizando el cálculo de áreas.

Por otra parte, un segundo tipo de respuesta fue puesto de manifiesto por 5 maestros en formación quienes realizan explicaciones directas del algoritmo de binomios conjugados, ya que articulan que una estrategia adecuada es mediante la exposición de $(a+b)(a$ $-b)=a^{2}-b^{2}$ (PF14). El tercer tipo de explicación refiere a la comparación entre el binomio al cuadrado y el binomio conjugado, ya que 4 profesores en formación afirman que sería congruente y adecuado enseñar el tema de binomios conjugados comparando las estructuras entre ambos y visualizando los resultados que se obtienen, de tal manera que después no se repitan dichas concepciones erróneas (PF5).

PF14: "Binomios conjugados son los que tienen los mismos términos, pero con diferente operación, es decir podemos tener $(x+7)(x-7)$ y el producto de ellos es $x^{2}-49$. Se coloca el cuadrado de cada uno de los términos".

PF5: "Haría una comparación de binomios cuando se tiene $(+y-)$ y $(+y+)$ ya que es importante que visualice que, aunque en ambos x se eleve al cuadrado y el 7 también, en el binomio $(x+7)(x+7)$ hay un término $14 x$, ya que no se elimina como sucede en el binomio $(x+7)(x-7)$ "'.

Finalmente, un total de 7 maestros en formación presentan inconsistencias respecto a la comprensión del producto notable. Cinco maestros en formación intentaron realizar explicaciones procedimentales, en estos casos los futuros profesores no identifican las diferencias entre el binomio al cuadrado y los binomios conjugados, además de que involucran términos y explicaciones erróneas. Por otro lado, 2 de los estudiantes para docente no contestaron al ítem.

En la tarea 4, en su ítem $b$ se cuestiona, ¿cómo le explicarías al alumno la simplificación de fracciones en casos donde se involucren productos notables?, se pone en manifiesto el diseño de instrucción del tema de simplificación de fracciones, para el cual se identificaron 3 distintas formas de realizar explicaciones de corte procedimental. El primer tipo de explicación fue manifestado por 10 estudiantes para maestro, el cual consiste en la eliminación de términos por medio de la factorización de productos notables. Un ejemplo claro de esto es el profesor en formación 16 que menciona lo siguiente. 
PF16: "Primero le pediría que analizara un poco su fracción y que observe si alguna expresión es parecida a algunos de los productos notables. Cuando lo observe realice la factorización conveniente y vea si existe una factorización viable, hasta reducir a su mínima expresión”.

El segundo tipo de explicación es la propuesta de 3 estudiantes para docente sobre la simplificación de fracciones por medio del desarrollo de la división (PF10). Por último, el tercer tipo de explicación es la expuesta por otros 3 maestros en formación que consiste en la repetición de ejercicios (PF11).

PF10: "Tal vez dividiendo sería una buena forma de enseñar simplificación".

PF11: "Realizando varios ejercicios para que a los mismos alumnos se les facilite observar cuando son productos notables, pues con la práctica se les hará más fácil el tema".

Finalmente, 14 estudiantes para profesor presentaron una mayor dificultad para abordar esta tarea. Siete futuros docentes no comprenden el tema y no saben cómo explicarlo, por lo que mencionan que la explicación adecuada para los alumnos es por medio de estrategias, reglas y ejemplos visuales; aunque no proporcionan ejemplo alguno. Asimismo, 7 no contestan a la interrogante.

La tarea 6 conlleva al análisis del conocimiento necesario para discriminar los ejemplos que se presentan al inicio del tema y para profundizar en él. En este sentido, 4 profesores en formación identifican que el ejercicio propuesto para iniciar puede generar dificultades para generalizar, por lo que mencionan que es recomendable únicamente, cuando los alumnos ya conocen el algoritmo. No obstante, este puede ser utilizarlo como demostración
(EP7). Por el contrario, 14 futuros docentes mencionan que, si es recomendable utilizar el ejercicio aritmético como ejemplo para iniciar el tema, debido a que dicho ejercicio es más fácil para los alumnos (PF20).

PF7: "No, ya que se tienden a usar solo números y al usar términos algebraicos se les complicaría. Se puede usar al final como demostración".

PF20: "Sí, porque es más fácil para ellos realizar una suma que empezar con multiplicaciones que involucren letras. También, enseñarle la jerarquía de operaciones y las multiplicaciones al cuadrado".

De igual forma, 6 maestros en formación no recomiendan el ejercicio para ser presentado al inicio del tema, aunque los argumentos empleados son inconsistentes (PF19). Como podemos visualizar, los profesores en formación dejan de lado el objetivo principal del tema que es la comprensión del producto notable (binomio al cuadrado), dándole principal importancia únicamente, a la facilidad en resolver algún ejercicio. Asimismo, 4 de 30 siguen la misma línea de respuesta respecto a que el ejercicio es recomendable, con la diferencia a que estos alumnos argumentan que a partir de la comparación del algoritmo y de los errores que se pueden cometer, el alumno puede aprender el tema significativamente (PF9).

PF19: "No es recomendable ya que el alumno se confundiría para la realización de binomios elevados a cualquier potencia".

PF9: "Para que el alumno cometa el error de sacarle el cuadrado a cada término y sumarlo, al ver que el resultado no es el mismo aprende del error". 
De acuerdo con el análisis de los ítems correspondientes a este conocimiento KCT podemos mencionar que la mayoría de los docentes en formación tiene el estatus de este conocimiento parcialmente desarrollado. Lo cual indica que se presentan debilidades para abordar el desarrollo del tema, desde la selección de ejercicios hasta el uso de diversas representaciones.

\section{CONCLUSIONES}

De manera global, los futuros profesores de matemáticas presentan un conocimiento limitado en cada uno de los subdominios del MKT. En el conocimiento común del contenido matemático presentan un mejor manejo de la estructura del producto notable, cuando este se presenta de manera descontextualizada. En el caso en el que el producto notable se contextualizó en un escenario de uso en los límites aparentemente indeterminados, el reconocimiento representó un mayor reto.

En el conocimiento especializado del contenido que refiere a las explicaciones, las representaciones, los procedimientos y los conceptos involucrados en este caso con los productos notables, también se identificaron inconsistencias. Particularmente, las explicaciones que justifican el método de resolución aritmética del producto notable resultaron superficiales en los maestros en formación. Por otro lado, la predicción, la identificación y la explicación de los errores que forman parte del conocimiento de contenido y los estudiantes resultaron ser insustanciales al referir a descripciones de los procedimientos y no a la fuente del error. Finalmente, respecto al conocimiento del contenido matemático y la enseñanza, los maestros en formación aluden a ejemplos de carácter procedimental evadiendo los de tipo conceptual.
Es importante mencionar que la finalidad no es enfatizar en las falencias de los futuros profesores, sino contextualizar que la formación que reciben tiene una incidencia directa en cómo interpretan y desarrollan las actividades matemáticas. De esta manera, las tareas y las actividades que se plantean a los docentes (durante su formación inicial e incluso continua) estructuran su conocimiento matemático para la enseñanza.

En el estudio que se desarrolla en este artículo, se refirió al MKT como un elemento teórico que puede guiar la formación del profesorado de matemáticas. Sin embargo, no es que se sostenga que el modelo MKT sea el único que puede aportar a dicha formación, pero es de importancia considerar los aportes que desde este modelo de conocimiento se propone, así como de las otras aportaciones realizadas desde la investigación y otros modelos del conocimiento del profesor, debido a que pueden estructurar una formación acorde a los objetivos de la educación matemática para los futuros docentes.

\section{RECONOCIMIENTOS}

El presente artículo de investigación se realizó en el marco del proyecto SEP-PRODEP DSA/103.5/15/10869.

\section{DECLARACIÓN DE LA CONTRIBUCIÓN DE LOS AUTORES}

El porcentaje total de contribución para la conceptualización, preparación y corrección de este artículo fue el siguiente: J. A. G. B. 40 \% y L. P. A. T $60 \%$. 


\section{DECLARACIÓN DE DISPO- NIBILIDAD DE LOS DATOS}

Los datos que respaldan los resultados de este estudio serán puestos a disposición por el autor correspondiente [L. P. A. T.], previa solicitud razonable.

\section{REFERENCIAS}

Ball, D. L.; Lubienski, S. \& Mewborn. D. (2001). Research on Teaching mathematics: The unsolve problema of teachers' mathematical knowledge. In V. Richarson (Ed.), Hanbook of research on teaching, Vol. 4 (pp. 433-456). New York, USA: Macmilian.

Ball, D. L.; Thames, M. H. \& Phelps, G. (2008). Content knowledge for teaching. What makes it special? Journal of Teacher Education, 59(5), 389-407. https://doi. org/10.1177/0022487108324554

Chang, C. \& Tsai, Y. (2005). An alternative Approach for the Learning of $(\mathrm{a}+\mathrm{b}) 2=\mathrm{a} 2+2 \mathrm{ab}+\mathrm{b} 2$. The 3 er East Asia Regional Conference in $\mathrm{Ma}$ thematics Education. Conferencia presentada en: The National Changhua University, Shanghai. Retrieved from http://ir.ncue.edu.tw/ir/ handle/987654321/14569

Creswell, J. W. (2009). Research Design: qualitative, quantitative, and mixed methods approaches (3. ed.). Londres: Sage

Dolores, C. (2013). La formación de profesores de matemáticas en México desde el currículum oficial. En C. Dolores, M. García, J. Hernández y L. Sosa (Eds.) Matemática educativa: la formación de profesores (pp. 13-25). Guerrero, México: Díaz de Santos Ediciones, S. A.

Godino, J. D. (2009). Categorías de análisis de los conocimientos del profesor de matemáticas. Revista Iberoamericana de Educación Matemática, 20, 13-31. Retrieved from https:// www.ugr.es/ jgodino/eos/JDGodino $\% 20$ Union_020\%202009.pdf

Gómez-Chacón, I. (2005). Tendencias y retos en formación de profesores en Matemáticas. Vivir el presente y crear futuro en la cooperación Europa-Latinoamérica. En I. Gómez-Chacón y E. Planchart (Eds.), Educación matemática y formación de profesores (pp. 15-32). Bilbao, España: Universidad de Deusto.

Graciano J. \& Aké L. P. (2017). Conocimiento común y especializado de productos notables de los futuros profesores de matemáticas. En Serna, Luis Arturo (Ed.), Acta latinoamericana de matemática educativa (pp. 13201329). México: Comité Latinoamericano de Matemática Educativa. Retrieved from http:// funes.uniandes.edu.co/12359/1/Graciano2017Conocimiento.pdf

Graciano J. \& Aké L. P. (2019). Conocimiento matemático para la enseñanza de productos notables: un estudio de tres casos. Revista Electrónica Investigación e Innovación en Matemática Educativa, 4(2)-Número especial, 192-201. Retrieved from http://revistaiime.org/index.php/IIME/article/view/55/20

Hill, H. C.; Ball, D. L. \& Schilling, S. G. (2008). Unpacking Pedagogical Content Knowledge Conceptualizing and Measuring Teachers' Topic-Specific Knowledge of Students. Journal of Research in Mathematics Education, 39(4), 372 - 400.

Hill, H. C.; Rowan, B. \& Ball, D. L. (2005). Effects of Teachers' Mathematical Knowledge for Teaching on Student Achievement. American educational research journal, 42(2), 371- 406. https://doi.org/10.3102/00028312042002371

León, O. G. \& Montero, I. (2003). Métodos de investigación en psicología y educación. ( $3^{\mathrm{a}}$ ed.). Madrid: McGraw-Hill.

López, E. (2008). Productos notables, factorización $y$ ecuaciones de segundo grado con una incógnita, una propuesta didáctica para el bachillerato del colegio de ciencias y humanidades. (Tesis de maestría). Facultad de Estudios Superiores Acatlán, Universidad Nacional Autónoma de México, México. Retrieved from https://repositorio.unam.mx/ contenidos/76286

Ma, L. (1999). Knowing and teaching elementary mathematics: teachers' understanding of fundamental mathematics in China and the United States. Mahwah, NJ: Erlbaum.

Navarrete-Cazales, Z. (2015). Formación de profesores en las Escuelas Normales de México. Siglo XX. Revista historia de la educación latinoamericana, 17(25), 17-34. https://doi. org/10.19053/01227238.3805 
Sosa, L. (2011). Conocimiento matemático para la enseñanza en bachillerato: un estudio de dos casos. (Tesis doctoral). Facultad de Educación, Universidad de Huelva, España. Retrieved from http://hdl.handle.net/10272/4509

Tzur, R. (2018). Profound awareness of the learning paradox (PALP). In B. Jaworski and T.Wood (Eds.), The Mathematics Teacher Educator as a Developing Professional, (pp. 137156). Rotterdam, The Netherlands: Sense Publishers.

Vega, D. C. (2013). Perfiles de alumnos de educación secundaria relacionados con el sentido estructural manifestado en experiencias con expresiones algebraicas. (Tesis doctoral).
Facultad de Educación, Universidad de Granada, España. Retrieved from http://hdl.handle.net/10481/31311

Vega-Gil, L. (2005). Los sistemas educativos europeos y la formación de profesores. Los casos de Francia, Reino Unido, España y Finlandia. Revista de Educación, 336, 169-187. Retrieved from http://www.revistaeducacion. mepsyd.es/re336/re336_10.pdf

$\mathrm{Wu}, \mathrm{H}$. H. (2018). The content knowledge mathematics teachers need. Mathematics Matters in Education (pp. 43-91). Springer, Cham. doi: https://doi.org/10.1007/978-3-319-61434-2_4

\section{(c) $(1)\left(3 \mathrm{BY} \bigodot_{\mathrm{ND}}\right.$}

Conocimiento de profesores de matemáticas en formación sobre los productos notables (Judith Graciano-Barragan - Lilia P. Aké). Uniciencia is protected by Attribution-NonCommercial-NoDerivs 3.0 Unported (CC BY-NC-ND 3.0) 